\title{
Impacto da terapia anti-retroviral na magnitude da epidemia do HIV/AIDS no Brasil: diversos cenários
}

\author{
Impact of antiretroviral therapy on the \\ magnitude of the HIV/AIDS epidemic in Brazil: \\ various scenarios
}

Maria Tereza S. Barbosa 1

Claudio J. Struchiner 2, 3

\footnotetext{
1 Departamento de Matemática e Estatística, Universidade do Rio de Janeiro. Rua Frei Caneca 94, Rio de Janeiro, $R J$ 22270-000, Brasil. 2 Programa de Computação Científica, Fundação Oswaldo Cruz. Av. Brasil 4365, Antiga Residência Oficial, Rio de Janeiro, $R J$ 21045-900, Brasil. tereza@procc.fiocruz.br stru@procc.fiocruz.br 3 Instituto de Medicina Social, Universidade do Estado do Rio de Janeiro. Rua São Francisco Xavier 524, 7o andar, Bloco D. Rio de Janeiro, $R J$ 20559-900, Brasil.
}

\begin{abstract}
We applied the back-calculation method to estimate the magnitude of the HIV epidemic in Brazil, using the EM and EMS algorithms. Under certain assumptions regarding the behavior of infected patients towards combined antiretroviral therapy, we discuss five different scenarios applied to the Brazilian epidemic. Our objective was to illustrate the impact of combined antiretroviral treatment on the incubation period and thus on estimates of the size of the HIVinfected population, based on reported AIDS cases.

Key words HIV; Acquired Immunodeficiency Syndrome; Surveillance; Highly Active Anti-Retroviral Therapy

Resumo Neste trabalho, utilizaram-se os algoritmos EM e EMS aplicados ao método do Cálculo Retroativo para estimar a magnitude da epidemia do HIV no Brasil. Fazendo-se suposições a respeito do comportamento dos infectados, em relação à utilização da terapia combinada das drogas anti-retrovirais, construíram-se cinco cenários para a epidemia brasileira. O objetivo foi o de ilustrar os impactos que a utilização da terapia combinada das drogas anti-retrovirais possam estar tendo ou possam vir a ter na incubação do vírus e, por conseguinte, nas avaliações da epidemia realizadas a partir dos casos de Aids notificados.

Palavras-chave HIV; Síndrome de Imunodeficiência Adquirida; Vigilância; Terapia Anti-Retroviral de Alta Atividade
\end{abstract}




\section{Introdução}

O Brasil é o quarto país em casos de AIDS, com 203.353 notificações até dezembro de 2000 . Mais de 20 anos depois dos primeiros casos, ainda não se pode assegurar que a epidemia esteja em declínio no país. Existem vários indicadores que demonstram o efeito positivo do tratamento da AIDS no Brasil. Segundo um documento do Ministério da Saúde (MS, 2000), houve uma redução em $38 \%$ das mortes entre 95 e 97 e uma redução entre 60 a $80 \%$ na ocorrência dos principais processos oportunistas relacionados com imunodeficiência grave em pacientes HIV positivo.

No entanto, estudos realizados nos Estados Unidos e Europa mostram que o declínio da incidência da AIDS, bem como das mortes causadas por ela a partir de 1996 (Buvé \& Rogers, 1998; Fleming et al., 1998), pode estar refletindo diminuição da incidência do HIV, mas pode também estar refletindo a incubação mais longa do vírus causada pela utilização da terapia combinada das drogas anti-retrovirais. Esta terapia tem se mostrado eficaz para manter ou recuperar a qualidade de vida e aumentar a sobrevida dos que estão infectados, mas seus efeitos biológicos e sociais ainda não estão totalmente definidos.

O relatório da Joint United Nations Programme on HIV/AIDS (UNAIDS, 1999) chama a atenção para a possibilidade de a prática do sexo seguro entre os homossexuais dos países do Ocidente estar diminuindo, devido a um impacto negativo na motivação de mudança de comportamento, causado pelo sucesso da terapia.

No Brasil, o início da terapia é recomendado apenas para aqueles pacientes com manifestações clínicas do HIV ou com contagem de células T-CD $4+<350$ células $/ \mathrm{mm}^{3}$, ainda não fazendo, portanto, efeito na incubação do vírus. Mas, no futuro, espera-se que o tempo entre a infecção pelo HIV e o diagnóstico de AIDS aumente, uma vez que os esforços na pesquisa clínica têm sido o de definir as melhores estratégias terapêuticas para o tratamento dos infectados pelo HIV (Schechter et al., 1999). Para realizar, com maior precisão, as projeções dos custos relacionados à distribuição gratuita destes medicamentos, é necessário estimar continuamente o tamanho da população portadora do HIV no Brasil, bem como a sua sobrevida esperada e o percentual dessa população que tomaria corretamente e com regularidade a terapia. Em relação às estimativas da população infectada no Brasil, o relatório da UNAIDS apontava 390 mil pessoas entre 15 e 49 anos no ano de 1997. Castilho (1997) estimou 549 mil infecta- dos até 1997 nesta mesma faixa etária e Szwarcwald \& Castilho (2000) estimaram 537 mil infectados em 1998. São diversos os métodos estatísticos e matemáticos aplicados com o objetivo de estimar a magnitude da epidemia do HIV e de fazer previsões sobre os casos atuais e futuros de AIDS. Eles se baseiam nas prevalências obtidas por pesquisas sentinelas realizadas com populações específicas ou partem das notificações oficiais de AIDS e fazem suposições a respeito da distribuição de incubação do vírus. Suas aplicações repetidas no tempo, incorporando novos aspectos da realidade, têm servido também como instrumento de reflexão a respeito de uma epidemia em mutação que está a cada momento mostrando uma nova face.

Uma reflexão necessária neste momento diz respeito ao entendimento do quanto e de que forma as notificações de AIDS podem continuar a servir como um espelho, sinalizando os efeitos dos tratamentos e das medidas de prevenção para os que planejam a política de sáude pública. Constroem-se, aqui, alguns cenários para a epidemia brasileira, a partir da inclusão de hipóteses a respeito dos efeitos que a distribuição gratuita da terapia anti-retroviral poderia estar tendo nas notificações de AIDS, nas estimativas do número de infectados pelo HIV e nas avaliações do comportamento da epidemia realizadas a partir destas notificações. Utilizou-se a metodologia descrita em Becker et al. (1991) que se baseia no método do cálculo retroativo desenvolvido por Brookmeyer \& Damiano (1989).

\section{Métodos}

A epidemia completa está representada na Tabela 1, onde os $\mathrm{Y}_{\mathrm{ij}}{ }^{\mathrm{j}}$-i são os infectados pelo HIV em $\mathrm{i}$, diagnosticados com AIDS em $\mathrm{j}$, onde $\mathrm{i} \leq \mathrm{j}$ e com d períodos de incubação, ou seja, $d=j-i$. Observe-se que, em cada tempo j, tem-se

$\mathrm{Y}_{\cdot \mathrm{j}}=\sum_{i=1}^{t} \mathrm{Y}_{i j}^{j-i}$

onde o tempo da infecção i varia do início da epidemia até um tempo t que, no máximo, é igual ao tempo j, quando o período de incubação for igual a zero. Os $\mathrm{Y}_{\mathrm{ij}}{ }^{+(\mathrm{j}-\mathrm{i})}$ representam os infectados pelo HIV em i que ainda não tiveram tempo de desenvolver os sintomas da AIDS até o momento L do estudo. Assim, na tabela, os $\mathrm{Y}_{\mathrm{j}}$ são os diagnosticados com AIDS em cada j e são as únicas observações contidas no Sistema de Informações de Agravos de Notificação (SINAN), uma vez que não se conhece o tempo de infec-

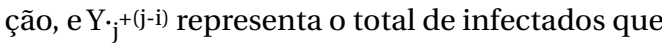
vão ser diagnosticados em um tempo j no futu- 
Eventos definidores de uma epidemia completa.

\begin{tabular}{|c|c|c|c|c|c|c|c|}
\hline \multirow[t]{2}{*}{ Infecção } & \multicolumn{5}{|c|}{ Diagnóstico } & & \multirow[t]{2}{*}{ HIV } \\
\hline & 1 & 2 & 3 & $\cdots$ & L & $\mathrm{L}^{+1}$ & \\
\hline 1 & $Y_{11}{ }^{0}$ & $Y_{12^{1}}$ & $Y_{13^{2}}$ & $\ldots$ & $Y_{1} L^{L-1}$ & $Y_{1(L+1)}+L$ & $N_{1}$ \\
\hline 2 & $\diamond$ & $Y_{22^{0}}$ & $Y_{23^{1}}$ & $\ldots$ & $\mathrm{Y}_{2 \mathrm{~L}^{\mathrm{L}-2}}$ & $Y_{2(L+1)}+(L-1)$ & $\mathrm{N}_{2}$ \\
\hline . & & & & & & & - \\
\hline . & $\diamond$ & $\diamond$ & $\nabla$ & $\cdots$ & $\cdots$ & $\cdots$ & $:$ \\
\hline . & & & & & & & \\
\hline $\mathrm{t}$ & $\diamond$ & $\diamond$ & $\Delta$ & $\ldots$ & $Y_{\mathrm{tL}} \mathrm{L}^{\mathrm{t}}$ & $Y_{t(L+1)}+(L+1-t)$ & $\mathrm{N}_{\mathrm{t}}$ \\
\hline AIDS & $Y_{\cdot 1}$ & $Y_{\cdot 2}$ & $Y_{3}$ & $\ldots$ & $Y_{\cdot L}$ & $Y_{\cdot(L+1)^{+}}$ & $N$ \\
\hline
\end{tabular}

ro. Os zeros estruturais, indicados como $\diamond$, representam a impossibilidade de o período de diagnóstico ser anterior ao da infecção.

O método do Cálculo Retroativo (Brookmeyer \& Damiano, 1989) parte da suposição de que o número de casos diagnosticados de AIDS até t, que é conhecido, pode ser_expresso como uma convolução do número de infectados a cada tempo e do período de incubação. Esta hipótese faz com que as inferências em relação à epidemia possam ser realizadas condicionalmente aos casos diagnosticados e a um padrão que relacione estas observações com os casos não observados de infectados pelo HIV. Este padrão estabelecido, por exemplo, ao se fazer hipóteses a respeito da densidade de infecção e a respeito da distribuição de incubação $\mathrm{f}_{\mathrm{d}}$ do vírus, que dá a probabilidade de se ter d períodos de incubação. Para as estimativas, Becker et al. (1991) utilizaram o algoritmo EM desenvolvido por Dempster et al. (1977), que formaliza uma antiga abordagem de manusear dados perdidos. Ao aplicálo neste contexto, deve-se, a partir de valores iniciais supostos para $\lambda_{1}, \lambda_{2}, \lambda_{3} \ldots \lambda_{\mathrm{t}}$, as taxas de infecção em cada t, substituir os valores perdidos (número de infectados não observados) pela esperança do número de infectados e, na etapa seguinte, atualizar as estimativas dos parâmetros, a partir da maximização da verossimilhança. Estas duas etapas repetem-se inúmeras vezes até a convergência das estimativas, definida utilizando a distância obtida em duas etapas consecutivas. Assim, a partir da suposição de que $\mathrm{N}_{1}, \mathrm{~N}_{2}, \ldots . \mathrm{N}_{\mathrm{t}}$, número não observado de infectados pelo HIV em cada t, são variáveis de Poisson independentes com médias $\lambda_{1}, \lambda_{2}, \ldots \lambda_{t}$, temos que $\mathrm{Y}_{\cdot_{1}}, \mathrm{Y}_{{ }_{2}}, \ldots . \mathrm{Y}_{{ }_{\mathrm{L}}}$, os casos diagnosticados de AIDS em cada j, também serão variáveis independentes de Poisson,
$\mathrm{E}\left(Y_{\cdot \mathrm{j}} \mid \mathrm{N}_{1}, N_{2}, \ldots, N_{t}\right)=\sum_{i=1}^{t} N_{i} f_{j-i}$

Em conseqüência, a esperança não condicional $E(Y . j)$ fornece o número médio de casos clínicos de AIDS em j será:

$\mu_{j}=\sum_{i=1}^{t} \lambda_{i} f_{j-i}$

e o logaritmo da verossimilhança será escrito como:

$\log L(\mathrm{~N}, \lambda ; \mathrm{Y})=\log L(\lambda ; \mathrm{Y})=\sum_{j=1}^{L}\left[\left(\sum_{i=1}^{j} \lambda_{i} f_{j-i}\right)^{y_{j}}-\sum_{i=1}^{j} \lambda_{i} f_{j-i}\right]$

onde $y_{\mathrm{j}}$ representa o total de diagnósticos de AIDS em j.

Para maximizar este função com a restrição de que o número de infectados não se altere muito em períodos vizinhos, utilizou-se o algoritmo suavizado (EMS) descrito por Becker et al. (1991). Neste, inclui-se, em cada etapa do algoritmo, uma etapa de suavização especificada por médias ponderadas de $\mathrm{k}$ períodos vizinhos a t, onde k é um número inteiro:

$\lambda_{t+i-k / 2}=\sum_{i=0}^{k} w_{i} \lambda^{n}$

Os pesos $\mathrm{w}_{\mathrm{i}}$ são simétricos e escolhidos a partir de suposições a respeito da realidade da epidemia e os $\lambda$ n são obtidos a cada etapa do algoritmo. Aqui, utilizaram-se os pesos binomiais:

$w_{i}=\left(\begin{array}{l}k \\ 2\end{array}\right) 2-k$ 


\section{Suposições a respeito do comportamento dos infectados}

Realizaram-se as estimativas a partir da suposição de que os infectados podem ter perfis diferentes em relação ao início e adesão ao tratamento e de que isto pode afetar a incubação do vírus. Na Figura 1, descrevem-se esquematicamente os efeitos na incubação relacionados a três possíveis comportamentos; (i) um primeiro grupo que só iniciou a terapia combinada após o diagnóstico de AIDS e, portanto, para esses não houve mudança no tempo de incubação (ii) um segundo grupo representado por aqueles que têm um tempo de incubação aumentado por tomar a terapia combinada por conta própria e com um alto grau de adesão; (iii) um terceiro grupo que teve o diagnóstico de AIDS apressado para ter acesso aos medicamentos gratuitos da rede pública, ou realmente teve o seu tempo de incubação reduzido por não ter tido um grau satisfatório de adesão aos medicamentos.

\section{Inclusão das suposições no modelo}

Becker \& Marschner (1993) demonstraram que incluir no modelo a informação a respeito dos casos de AIDS em cada tempo e por idade no

Figura 1

Desenho esquemático dos comportamentos relacionados à terapia.

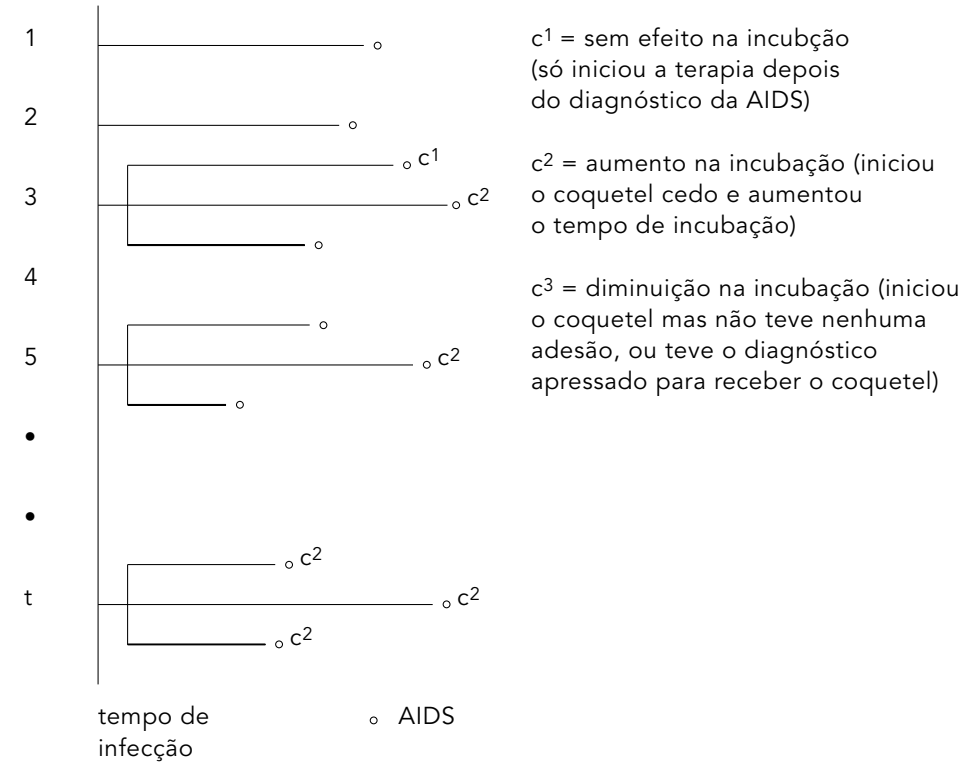

diagnóstico torna mais precisas as estimativas da incidência do HIV e permite estimar a suscetibilidade relativa por idade.

Para incorporar, além da idade no diagnóstico, os perfis de infectados segundo seu comportamento em relação à terapia combinada, bem como seus efeitos na incubação do vírus, substituiu-se a distribuição $\mathrm{f}_{d}$ por uma incubação "ponderada" pelas proporções de infectados em cada comportamento, transformada de acordo com os efeitos que os comportamentos exercem na incubação e dependente do período de infecção.

Assim, as suposições de que existem três comportamentos distintos em relação à terapia combinada, que a proporção de infectados com cada comportamento é $\mathrm{p}_{1}, \mathrm{p}_{2}$ e $\mathrm{p}_{3}$ e que cada comportamento modifica a incubação segundo as taxas $\mathrm{q}_{1}, \mathrm{q}_{2}$ e $\mathrm{q}_{3}$, respectivamente, modifica a equação de convolução de uma epidemia completa para:

$\mathrm{E}\left(Y_{a j} \mid\left\{\mathrm{N}_{\mathrm{at}}\right\}\right)=\sum_{i=1}^{3} \sum_{i=1}^{t} p_{x} N_{a i} f_{j-i, x}$

onde $\mathrm{Y}_{\mathrm{aj}}$ é o número observado de casos de AIDS diagnosticados na idade a no tempo j, $\mathrm{N}_{\text {at }}$ é o número não observado de indivíduos na idade a infectados pelo HIV no tempo t e $f_{j-i}, x$ é a distribuição de incubação para quem se infectou em i e teve o comportamento x. Para um período t fixo,

$$
\begin{aligned}
& \sum_{x=1}^{3} N_{a t} f_{j-t, x}=p_{1} N_{a t} q_{1} f_{j-t}+p_{2} N_{a t} q_{2} f_{1-2}+ \\
& +p_{3} N_{a t} q_{3} f_{j-t}=N_{a t} f_{j-t}\left(p_{1} q+p_{2} q_{2}+p_{3} q_{3}\right)= \\
& =N_{a t} g_{j-t}
\end{aligned}
$$

e para estimar os valores de $\mathrm{N}_{\mathrm{at}}$, o modelo considerado será:

$\mathrm{E}\left(Y_{a j} \mid \mathrm{N}_{\mathrm{a} 1}, N_{\mathrm{a} 2}, \ldots, N_{\mathrm{a} t}\right)=\sum_{i=1}^{t} v_{(a-j+i) i} g_{i(j-i)}$,

onde $g_{i(j-i)}$ é a incubação ponderada e transformada conforme a equação (1), $\mathrm{N}_{\mathrm{a} 1}, \mathrm{~N}_{\mathrm{a} 2}, \ldots, \mathrm{N}_{\mathrm{at}}$ são variáveis de Poisson independentes, $\mathrm{E}_{(\mathrm{Nat})}=$ $\mathrm{v}_{\mathrm{at}}, \mathrm{E}_{(\text {Yaj) }}=\mu_{\mathrm{aj}}$.

A dependência da idade foi incorporada fazendo-se $\mathrm{v}_{\mathrm{at}}=\pi_{\mathrm{a}} \beta_{\mathrm{a}} \lambda_{\mathrm{t}}$, onde $\pi_{\mathrm{a}}$ é a proporção de indivíduos da população na idade a e $\beta_{\mathrm{a}}$ reflete a suscetibilidade relativa da idade a.

Visando a resolver problemas de identificabilidade, foi imposta a restrição

$$
\sum_{a=1}^{A} \pi_{\mathrm{a}} \beta_{\mathrm{a}}=1 \text {. }
$$

Com esta restrição, a estimativa de $\lambda_{t}$ não dependerá da idade e poderá ser comparada com os resultados anteriores. 
Considerando que os dados completos seriam os $\mathrm{N}_{\mathrm{a} t \mathrm{~d}}$, o número de indivíduos de idade a infectados até t com duração de incubação d, a verossimilhança maximizada para $\mathrm{N}_{\mathrm{atd}}=\mathrm{n}_{\mathrm{atd}}$ foi:

$\log L\left(\beta, \lambda \mid\left\{n_{\text {aid }}\right\}\right)=$

$=\sum_{i=1}^{t} \sum_{a=1}^{A} \sum_{d=0}^{L-i}\left\{n_{\text {aid }} \log \left(\beta_{a} \pi_{a} \lambda_{\mathrm{t}} f_{i d}\right)-\pi_{a} \beta_{a} \lambda_{\mathrm{t}} f_{i d}\right\}$

O passo E (Esperança) do algoritmo EM consistiu em substituir $\mathrm{n}_{\mathrm{atd}}$ por

$\hat{\mathrm{n}}_{\mathrm{aid}}=\mathrm{E}\left(\mathrm{N}_{\mathrm{aid}} \mid y ; \beta\right.$ velho, $\lambda$ velho $)=$

$=y_{a+d, i+d} \frac{\pi_{a} \beta_{a} \lambda_{i} f_{i d}}{\sum_{d=0}^{L-i} \pi_{a+i-j} \beta_{a+i-j} \lambda_{\mathrm{i}} f_{i, i+d-i}}$

O passo M (Maximização) consistiu em maximizar a nova verossimilhança com respeito a $\beta$ e a $\lambda$.

A etapa da suavização neste caso incluiu ponderações e número de períodos para os parâmetros referentes a infecção pelo HIV e à suscetibilidade ao vírus por idade e:

$\beta_{a^{\text {novo }}}=\sum_{i=0}^{k_{1}} w_{i 1} \beta^{*}{ }_{a+i-k_{1} / 2}$

$\lambda_{t}^{\text {novo }}=\sum_{i=0}^{k_{2}} w_{i 2} \lambda^{*}{ }_{t+i-k_{2} / 2}$

Onde $K_{1}$ e $k_{2}$ são os períodos de suavização para a infecção e para a suscetibilidade por idade e $\mathrm{w}_{1}$ e $\mathrm{w}_{2}$ sâo os respectivos ponderadores. Para determiná-los, considerou-se que a necessidade de suavização para a suscetibilidade por idade supõe comportamentos semelhantes entre adultos num intervalo etário de até dois anos, ao passo que a suavização para a infecção pelo HIV supõe comportamentos semelhantes em anos vizinhos. $\mathrm{O}$ algoritmo EM foi programado no S-PLUS.

\section{Dados utilizados}

Utilizaram-se os 153.696 casos de AIDS no Brasil diagnosticados entre janeiro de 1982 e dezembro 1998, notificados até fevereiro de 1999, com idade no diagnóstico compreendida entre 15 e 66 anos. Aos diagnósticos nos anos de 1993 a 1998, aplicou-se homogeneamente, para todas as idades, a correção de atraso proposta por Barbosa \& Struchiner (2002) obtendo-se, então, 191.392 casos.

A idade na infecção foi considerada entre 15 e 49 anos, de modo a se manter coerência com a idade no diagnóstico de AIDS compreendida entre 15 e 66 anos em um período de observação de 18 anos. Nesta faixa etária estão 99\% dos diagnósticos de AIDS em adultos no Brasil. Calculou-se a proporção da população brasileira em cada idade utilizando-se dados obtidos na Contagem Populacional realizada pela Fundação Instituto Brasileiro de Geografia e Estatística (IBGE, 1996). A distribuição de incubação utilizada foi a de Weibull com tempo de incubação mediano de nove anos, com parâmetros 0,098 e 2,516, e dez anos, com parâmetros 0,086 e 2,516 . Vale a pena observar que a escolha da família Weibull está associada a uma hipótese de que o risco do desenvolvimento de AIDS ao longo do tempo vai crescendo indefinidamente e a escolha dos parâmetros partiu daqueles que vêm sendo mais utilizados.

Construíram-se os cenários ao se variar a proporção dos infectados em cada um dos comportamentos e combinar com suposições a respeito dos efeitos destes comportamentos na incubação real ou aparente do vírus. Eles estão descritos na Figura 2.

\section{Resultados}

Na Tabela 2 podem-se observar as previsões de casos de AIDS para os anos de 1999 a 2001 e o número acumulado de infectados pelo HIV até 1998 nos cinco cenários. Estas foram as estima-

Figura 2

Desenho esquemático dos cenários.

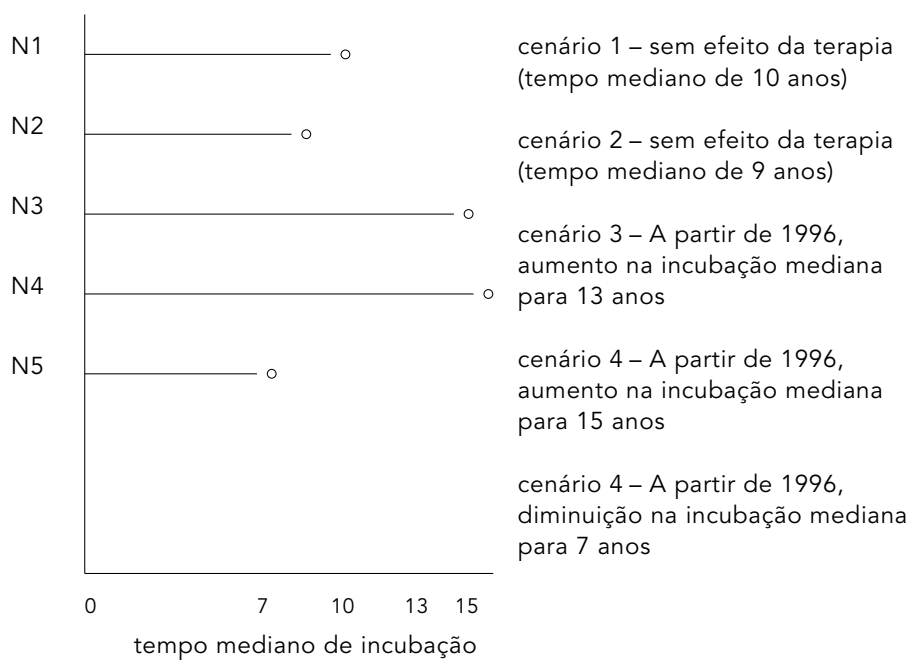


tivas encontradas ao se considerarem efeitos distintos do uso da terapia combinada na incubação, a hipótese de suavidade em períodos vizinhos, a ocorrência da infecção segundo uma distribuição de Poisson e o tempo de incubação seguindo uma distribuição Weibull.

As estimativas para o número de adultos já infectados pelo HIV desde o início da epidemia até o ano de 1998 no Brasil variaram de 648 mil, quando a suposição era de um tempo mediano de nove anos constante desde o início da epidemia, a 984 mil, ao supor uma incubação no início da epidemia com mediana de dez anos e o tempo de incubação aumentado em $50 \%$ a partir de 1996. As previsões dos diagnósticos de AIDS, a partir dos infectados estimados nos diversos cenários, também variaram para os diag-

Tabela 2

Estimativas do número acumulado de infectados pelo HIV no Brasil até 1998 e dos novos casos de AIDS.

\begin{tabular}{lllll}
\hline Cenários & HIV & \multicolumn{3}{c}{ AIDS } \\
& & 1999 & 2000 & 2001 \\
\hline 1 (básico) & 766.629 & 38.725 & 41.569 & 43.809 \\
2 & 648.683 & 36.437 & 38.599 & 40.002 \\
3 & 841.390 & 41.397 & 44.393 & 46.644 \\
4 & 984.285 & 45.381 & 48.895 & 51.505 \\
5 & 720.677 & 37.067 & 39.897 & 42.231 \\
\hline
\end{tabular}

Figura 3

Suscetibilidade estimada por idade.

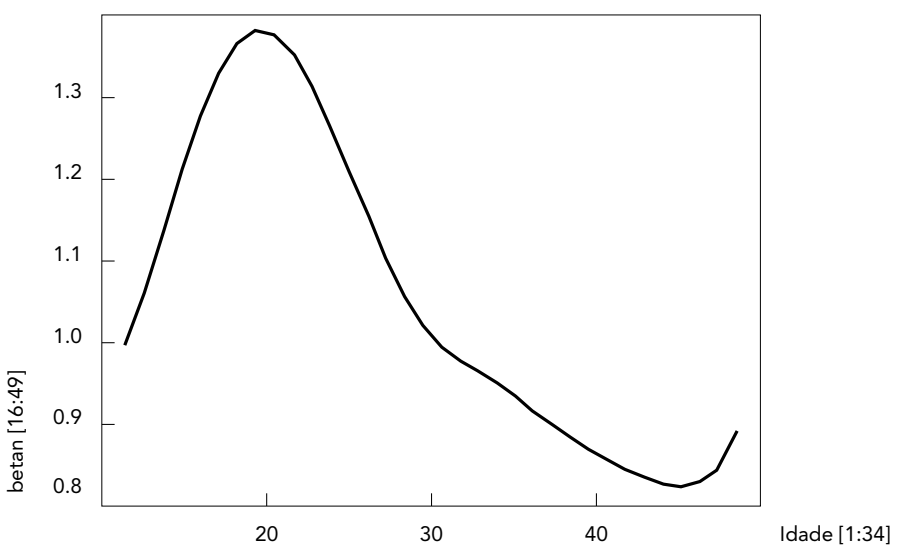

Cad. Saúde Pública, Rio de Janeiro, 19(2):535-541, mar-abr, 2003 nósticos em 1999, de 36.437 a 45.381 nos dois cenários descritos anteriormente.

As estimativas das suscetibilidades relativas por idade não diferiram muito entre si. Pode-se verificar, observando-se a Figura 3, que o risco relativo de infecção cresce a partir dos 13 anos, atinge o máximo logo após os 20 anos, decresce até os 40 , onde começa a crescer novamente.

\section{Discussão}

Utilizaram-se, aqui, as metodologias descritas em Becker et al. (1991) e Becker \& Marschner (1993), para avaliar possíveis impactos da introdução da terapia combinada na ocorrência das notificações da AIDS e nas estimativas do número de infectados pelo HIV no Brasil. Devemse avaliar estas estimativas considerando-se as limitações metodológicas, bem como as suposições feitas na construção dos cenários. Não existem ainda estimativas da distribuição de incubação para coortes brasileiras e, assim, além de todas as influências nas estimativas do método do Cálculo Retroativo já discutidas por Bacchetti et al. (1993) e Mariotti \& Cascioli (1996), acrescente-se também a incerteza por utilizarmos dados relativos a um outro país em que a epidemia deve ter características diferentes. Vale a pena lembrar que os cenários foram criados com o objetivo de ilustrar os efeitos que o aumento da incubação pode vir a ter em uma epidemia em que a avaliação de seu comportamento se dê principlamente a partir dos casos sintomáticos já notificados, do tempo de sobrevida após o diagnóstico de AIDS e da taxa de mortalidade. Eles podem auxiliar também a discussão a respeito dos recursos necessários para garantir que a maior parte dos infectados pelo HIV tenha acesso e boa adesão aos tratamentos disponíveis, de forma a prolongar ao máximo sua incubação e impedir que o uso dos medicamentos de forma irregular acelere o processo de seleção de cepas virais resistentes.

Pretende-se que essa discussão se contraponha a uma expectativa existente, de que a mudança de comportamento nas categorias de maior exposição ao risco de se contaminar seria o pré-requisito para o início do declínio da epidemia e que isto já estaria garantido no Brasil. Em relação às estimativas das suscetibilidades por idade, verificou-se que os cenários não diferiram muito entre si e apontaram para as mesmas faixas etárias com maior probabilidade de infecção e para onde devem estar sendo dirigidas as campanhas de prevenção. Deve-se considerar, no entanto, o potencial de disseminação do vírus na população pobre brasileira. É 
claro que, ao se obter estas estimativas por categoria de exposição, por sexo e por alguma variável de estratificação econômica, mais informações serão obtidas para auxiliar estas campanhas.

\section{Referências}

BACCHETTI, P.; SEGAL, M. R. \& JEWELL, N. P., 1993. Backcalculation of HIV infection rates. Statistical Science, 8:82-119.

BARBOSA, M. T. S. \& STRUCHINER, C. J., 2002. The estimated magnitude of AIDS in Brazil: A delay correction applied to cases with lost dates. Cadernos de Saúde Pública, 18:279-285.

BECKER, N. G. \& MARSCHNER, I. C., 1993. A method estimating the age-specific relative risk of HIV infection from AIDS incidence data. Biometrika, 80: 165-178.

BECKER, N. G.; WATSON, L. F. \& CARLIN, J. B., 1991. A method of non-parametric Back-Projection and its application to AIDS data. Statistics in Medicine, 10:1527-1542.

BROOKMEYER, R. \& DAMIANO, A., 1989. Statistical methods for short term projections of AIDS incidence. Statistics in Medicine, 8:23-34.

BUVÉ, A. \& ROGERS, M. F., 1998. Epidemiology. AIDS, 12(Sup. A):S53-S54

CASTILHO, E. A., 1997. Estimativas do número de pessoas entre 15 e 49 anos de idade infectadas pelo HIV. Brasil, 1996. In: A Epidemia da AIDS no Brasil: Situação e Tendências (Ministério da Saúde, org.), pp. 13-14, Brasília: Ministério da Saúde.

DEMPSTER, A. P.; LAIRD, N. M. \& RUBIN, D. B., 1977. Maximum likelihood from incomplete data via the EM algorithm. Journal of the Royal Statistical Society, Series B, 39:1-38.
FLEMING, P. L.; WARD, J. W.; KARON, J. M.; HANSON, D. L. \& DE COCK, K. M., 1998. Declines in AIDS incidence and deaths in the USA: A signal change in the epidemic. AIDS, 12(Sup. A):S55-S61.

IBGE (Fundação Instituto Brasileiro de Geografia e Estatística), 1996. Contagem Populacional. Rio de Janeiro: IBGE.

MARIOTTI, S. \& CASCIOLI, R., 1996. Sources of uncertainty in estimating HIV infection rates by backcalculation: An application to Italian data. Statistics in Medicine, 15:2669-2687.

MS (Ministério da Saúde), 2000. Recomendações para Terapia Anti-Retroviral em Adultos e Adolescentes Infectados pelo HIV. Brasília: MS.

SCHECHTER, M.; STRUCHINER, C. J. \& HARRISON, L. H., 1999. Protease inhibitors as initial therapy for individuals with an intermediate risk of HIV disease progression: Is more necessarily better? AIDS, 1:97-102.

SZWARCWALD, C. L. \& CASTILHO, E. A., 2000. Estimativa do número de pessoas de 15 a 49 anos infecta das pelo HIV, Brasil, 1998. Cadernos de Saúde Pública, 16(Sup. 1):135-141.

UNAIDS (Joint United Nations Programme on HIV/ AIDS), 1999. Epidemic Situation. UNAIDS Report. Geneva: World Health Organization.

Recebido em 15 de outubro de 2001

Versão final reapresentada em 20 de agosto de 2002 Aprovado em 2 de dezembro de 2002 